\title{
AMIOTROFIAS POR LESOES DO LOBO PARIETAL
}

\author{
JAIME OLAVO MARQUEZ *** \\ FRANCISCO MAURO GUERRA TERRA * \\ JOAO JOSE DE BARROS ** \\ EDSON BOUZA MARQUEZ***
}

A atrofia muscular como conseqüência de lesões do lobo parietal não é rara, havendo muitas publicações que detalham exaustivamente seu cotejo sintomático, sua etiologia e a importância como sinal clínico bastante precoce 1,2,3,14,18,19,21.

Este trabalho foi feito visando a realçar este achado neurológico, para o qual foi chamada bastante atenção no passado, quando Silverstein 18 insistia no exame meticuloso dos pacientes, julgando que havia negligência na semiótica, atribuivel a causas que, acreditamos, continuam motivando tal esquecimento ainda hoje (dificuldade para despir pacientes paralizados, displicência no exame clínico pela super-valorização dos exames laboratoriais, pouca atenção aos achados neurológicos no pós-operatório). Em cursos e congressos, e em revisões que fizemos em livros de textos utilizados rotineiramente na formação de neurologistas e neurocirurgiōes, com exceção de ligeiro tópico no Clinical Neurology (Baker \& Baker, 1976) não encontramos referência a este importante sinal de localização. Considerando que no espaço de um ano, trabalhando em serviço relativamente pequeno, conseguimos registrar três casos, julgamos válido este relato.

\section{OBSERVAÇOES}

Caso 1 - J. L. S. (Reg. 33096) internado em 08-01-1976. Paciente do sexo masculino, com 28 anos de idade, apresentando cefaléia, instalada dois meses antes. Ao exame neurológico, notou-se apenas edema de papila bilateral discreto; nenhum sinal de localizaç̆o. Como a reaçăo de Weinberg resultasse reagente no liquido cefalorraqueano, o diagnóstico inicial foi de neurocisticercose. Foi instituida a medicaçăo indicada, quando apos 5 dias de tratamento, ainda em um periodo de observaçă, fol solicitada a alta pelo coordenador do INPS. Dez dias após, retornou o paciente com o mesmo quadro anterior agravadio por discreta hemiparesia esquerda. Angiografia carotídea direita revelou processo expansivo parieto-temporal. Depols da intervençáo cirúrgica, desenvolveu-se intensa amiotrofia dos interósseos da måo esquerda, em um periolo de 6 dias. o diagnostico histopatológico da peça cirúrgica foi de astrocitoma grau III.

Caso 2 - A. P. S. (Reg. 30781) internado em 26-08-1975. Paciente do sexo masculino, com 59 anos de idade. Seus padecimentos iniciaram-se com frequentes episódios de desajuste familiar, três meses antes da internação, com rápidas manifestaçōes de deso-

Disciplina de Neurologia da Faculdade Federal de Medicina do Triangulo Mineiro (Uberaba, MG, Brasil): *Professor Titular; *Professor Assistente; **Auxilliares de Ensino. 
rientação temporo-espacial. Atendido por clínico geral, teve o diagnóstico de síndrome de insuficiência vascular cerebral. Dois meses após, apresentou episódio confusional, que acreditamos ter sido de desiorientaçăo em relaçăo ao esquema corporal (vestiu os dois membros inferiores em uma sర perna da calça). Ulteriormente, progressiva instalação de hemiparesia esquerda, cefaléia e abulia. Ao exame neurológico, encontramos o paciente contactuando mal, năo sendo possível a avaliaçăo da semiologia da sensibilidade, com hemiparesia esquerda e sinais de liberaçăo piramidal, edema de pápila bilateral, discreta amiotrofia da măe esquerda (interósseos dorsais) que năo foi devidamente valorizada. A angiografia carotídea mostrou processo expansivo parietal direito. O diagnóstico histopatológico da peça círúrgica foi de astrocitoma grau III. Desenvolveu-se, após a cirurgia, junto à discreta hemiparesia esquerda, rápida e intensa amiotrofia da măo esquerda em três dias (Fig. 1).

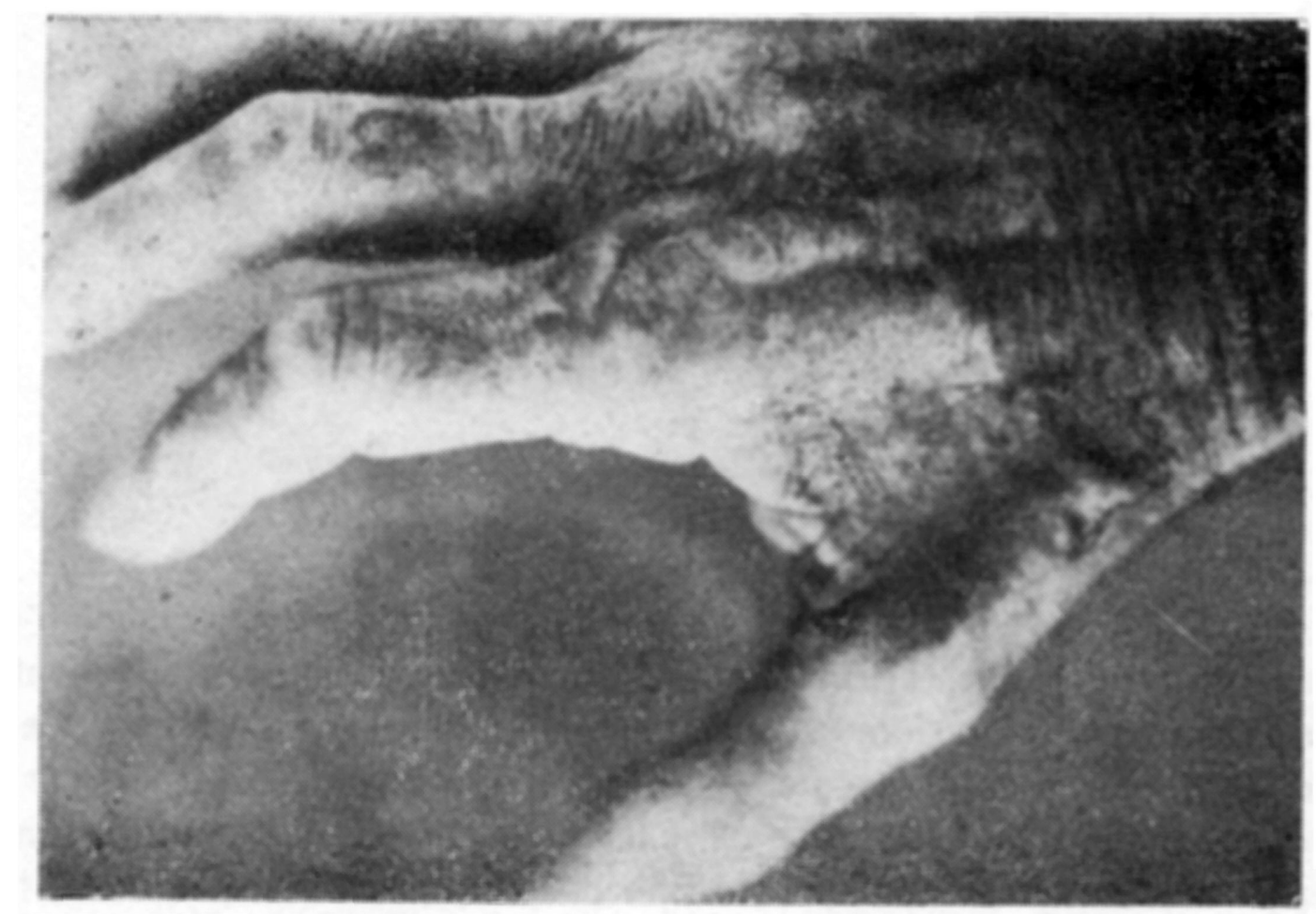

Fig. 1 - Caso A. P. 8. Aspecto da amiotrofia (10 interosseo dorsal)

Caso 3 - M.A.N.B. (Reg. 30076) internada em 02-11-1974. Paciente do sexo feminino, com história de, aproximadamente há três meses, estar apresentando manifestaç̋es da esfera emocional. Recebeu tratamento psiquiátrico durante dois meses, com poucos resultados. Quinze dias antes da internaçăo, apresentou crise de automatismo psicomotor. Ao exame neurológico a paciente estava indiferente ao meio e à sua condiça mórbida, reagindo pouco aos estímulos dolorosos, sem evidenciar comprometimento motor. Chamava a atençăo, alteraçăo trofica de ambas as măos que, segundo seus familiares, datava já de dois meses (Fig. 2). Ao exame de fundo de olho notou-se grande edema de papila bilateral. O eletroencefalograma mostrou lentificaçăo à direita. O exame angiografico carotídeo evidenciou processo expansivo parieto-occipital direito. Mediante craniotomia foi encontrado grande meningioma, medindo $10 \times 8 \mathrm{~cm}$. A paciente evoluiu bem no pós-operatório, notando-se logo melhora da situaçăo trófica e na mobilidade dos dedos de ambas as mãos. 


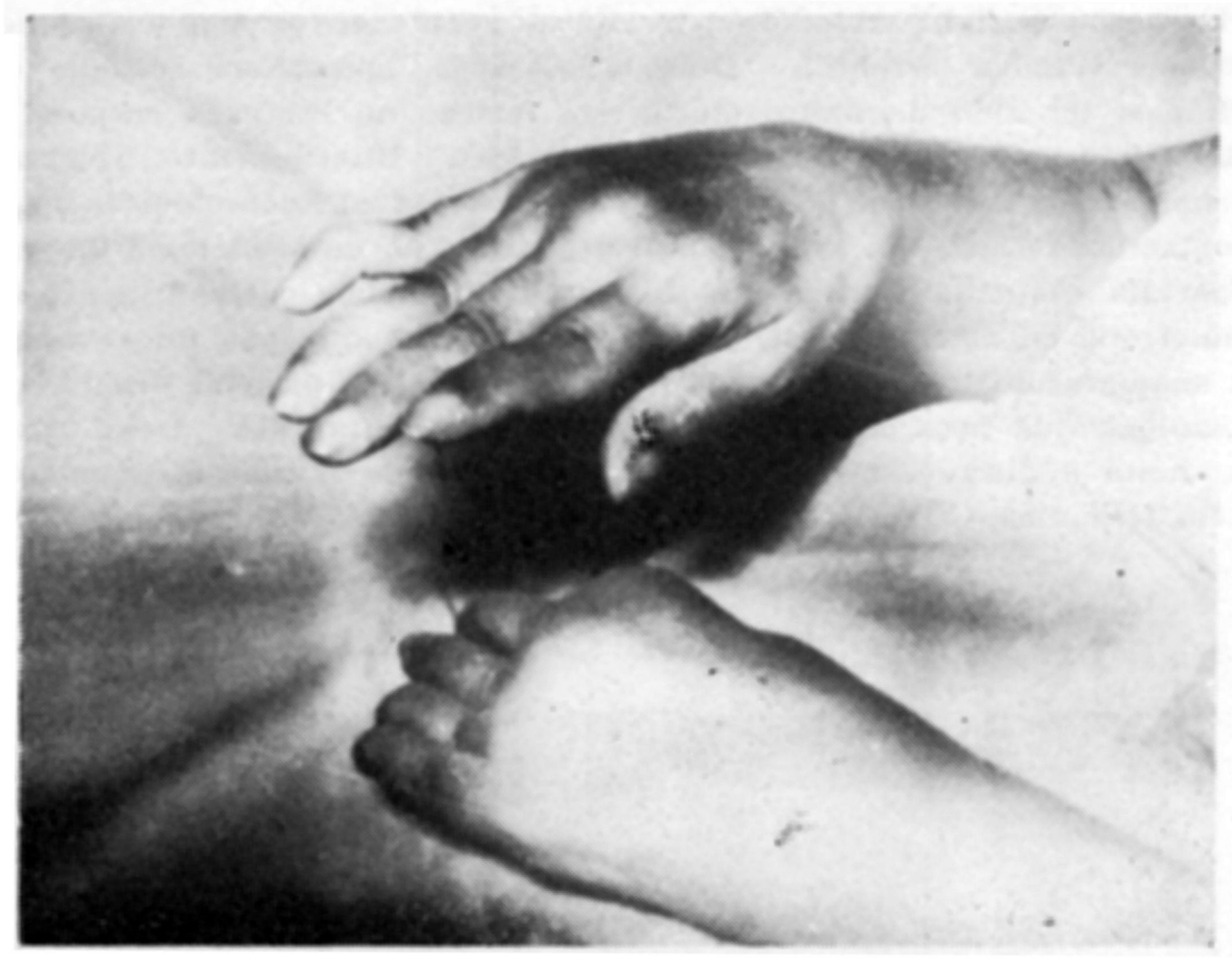

Fig. \& - Caso M. A. N. B. Aspecto das mäos

\section{COMENTARIOS}

Foi Silvestein 18 que enfatizou o valor diagnóstico de localização da amiotrofia que leva seu nome, afirmando ser este elemento bastante encontrado em pacientes em estado confusional, coma, ou com alterações sensitivas de modo a não poder informar fidedignamente. Silverstein e outros mostraram que o exame detalhado dos músculos e da pele de pacientes com lesões do lobo parietal ou de suas proximidades, revelou ser este tipo de amiotrofia extremamente frequente. Estas mesmas observações tinhm sido encontradas em relatos de Chatin (1900): e Steinert (1906) ${ }^{19}$, tendo Charcot 2 feito o primeiro estudo de importância sobre este assunto. Foi Prince (1906) ${ }^{16}$ quem demonstrou que intensa amiotrofia, simulando acometimento medular (até mesmo síndrome de Aran-Duchenne), pode ocorrer na fase inicial de tumores cerebrais. Tanto quanto as causas tumorais, os traumatismos também desencadeiam o referido quadro ${ }^{11.12,17}$.

Guthrie (1918)* alinhou, ao lado da amiotrofia contralateral, hipotonia, alterações sensitivas e os distúrbios tróficos da pele (ladylike hand), descrevendo os achados clínicos de maneira bem completa. Tais distúrbios tróficos são encontrados também nos acidentes vasculares cerebrais e na encefalopatia crônica da infância com lesões do lobo parietal, sendo neste caso regularmente associada a diminuição global de crescimento contralateral ${ }^{12}$. Silverstein 18 estudou 90 casos, sendo $11 \%$ de causa tumoral, $33 \%$ de encefalopatia crônica da infância e $55 \%$ de pacientes hemiplégicos em que o autor somente estabeleceu relação entre forma hipertônica e hipotônica e alterações sensitivas, não entrando em detalhes sobre sua etiologia (Grupo III de Silverstein). 
A fisiopatogenia é muito discutida. Charcot 2 atribuia a amiotrofia a uma esclerose descendente do feixe piramidal, com acometımento secundário das células da ponta anterior da medula. Apesar de Babinski ${ }^{1}$ ter demonstrado que tal atrofia poderia ser encontrada mesmo sem lesão da medula ou do neurônio motor periférico, durante muito tempo alguns autores continuaram impregnados com as idéias de Charcot. Outros acreditam que haveria um verdadeiro centro trófico no lobo límbico ou no lobo parietal ", intimamente relacionado à função sensorial. Delmas Marsalet ' mostrou a intima relação entre a perda sensitiva e a atrofia; em seus dois casos a atrofia progrediu proporcionalmente à intensidade de deficit sensitivo, melhorando paralelamente com 0 retorno sensitivo. Pitha, Masin e Polak ${ }^{13}$ diferem da opiniăo de Silverstein quanto à exclusividade do lobo parietal como desencadeante do processo amiotrófico. Sua participação seria marcante mas não absoluta pois, em 10 casos, assinalaram $20 \%$ com localização parieto-occipital e $20 \%$ na regiāo frontal.

Se haveria ou não lesão da vida piramidal, os autores 14.18 concordam que a mesma não é necessária para a instalação de amiotrofia central. Este fato toi demonstrado, com a publicaçao por sllverstein ${ }^{18}$, de dois casos de lesäo parietal, apenas com amiotrofia e crises tipo Bravais-Jacksonianas, sem qualquer outra manıtestação neurológica. As formas paralıtıcas flácidas são bastante trequentes, com tendência à espasticidaue progressiva em $2 / 3$ uos casos, acreuıtandu-se mesmo que a amıotrotıa dependerıa muıto da presença aa hipotonıa‘,1s. Estando intactas as vias eferentes (pıramidal e a extrapiramıal), supöe-se uma lesäo de aterencia do lobo parietal ou do lobo trontal, que inrluırı no tono muscular, principalmente no de repouso ${ }^{1+}$. Silverstein ${ }^{18}$ observou hipotonia e amiotrotia imediatamente apos intervençao cırurgica na regıao parıetal (algumas até 48 horas após), e em aciuentes vasculares ceı evra1s, até no quarto dia após 0 insulto, significando que a instalaçäo da amıtrotia ındepende da espastıcidade bem como da paresia proprıamente dita. $U$ desgaste do tecido muscular é mais intenso nas lesões corticais atetando tanto as vias motoras como vias sensitivas. Christiansen * chamou a atençao para a relaçäo existente entre 0 aparecimento de crises Bravais-Jacksonianas e a intensidade da amiotrofia nesta mesma região.

Quanto ao aspecto clínico, além de alguns dados já apresentados, poderiamos resumir os achados, em atrofia intensa contra-lateral, de instalação muito precoce, com hipotonia da região em sua fase inicial, com alterações sensitivas, ocorrendo mais no membro superior, junto com alterações tróficas da mão, tipo mão de dama (pálida, com perda das linhas dos dedos e macia à palpação). Nas alterações sensitivas, a mais encontrada é a estereoagnosia. Dana s atribui a responsabilidade da amiotrofia à um fenômeno que chamou de "toucher actif", significando que o trofismo muscular dependeria das aferências sensitivas, mais do que da integridade motora. Poderia ocorrer ainda, uma sindrome sensitiva tipo tabética (síndrome de Dejeirine-Verger) ou uma síndrome tipo siringomiélica (sindrome de Dejerine-Mouzon) ${ }^{15}$.

Para finalizar chamamos a atenção para o caso de $\mathrm{n}^{\circ} 3$, com alteração trófica bilateral, que não sabemos como explicar, e para o fato de que na bibllo. 
grafia consultada, encontramos referências à manifestações unicamente contralaterais à lesão.

Em conclusão, julgamos ser do maior interesse semiológico, o exame minucioso dos relevos musculares, particularmente das extremidades, nos pacientes com vários tipos de patologias intracranianas. Sempre que possível, devemos realizar semiologia cuidadosa das sensibilidades em geral.

\section{RESUMO}

E feita uma revisão bibliográfica sobre a amiotrofia de Silverstein, com aspectos históricos e fisiopatogênicos relativos às manifestações clínicas. Os autores discutem a possível causa das escassas referências sobre 0 assunto, e relatam três casos próprios, deixando em aberto a explicação para um deles que apresentou amiotrofia controlateral e do mesmo lado da lesão, melhorando com tratamento cirúrgico, fato não encontrado na revisão bibliográfica realizada.

\section{SUMMARY}

Amyotrophy by parietal lobe lesion: report of three cases.

A bibliographical revision about Silverstein's amyotrophy, with a historical sight analizing the physiopathogeny and its clinical manifestations is reported. The authors discussed the possible cause of the few references in the literature about the matter and relate three cases; they leave a blank in one of them with amyotrophy in the other side and the same side of the lesion, that got better with surgical treatment. This was not found in the bibliographical revision that has been looked for.

\section{REFERENCIAS}

1. AJURIaguerra, J. \& HeCAen, H. - Le Cortex Cérébrale: tude Neuro-PsychoPathologique. Masison et Cie., Paris, 1964.

2. CHARCOT, J. M. - Lectures on Diseases of the Nervous System (1879) Translated by Geo Sigerson. Henry C. Lea, Philadephia pg. 64.

3. CHATIN, P. - Troubles trophiques et troubles de la sensibilité chez les hemiplegiques. Rev. Med. 20:781, 1900.

4. Christiasen, K. V. - Les Tumeurs du Cerveau. Tese, Paris 1921.

5. DAVID, M.; HECAEN, H. \& SAUGUET, H. - Les amyotrophies d'origine parietale. Prèsse Med. 19:282, 1944.

6. DELMAS MARSALET, M. - Sur les atrophies musculaires d'origine parietale. Rev. Neurol. (Paris) 73:455, 1941.

7. GOWERS, W. R. - The brain in congenital abscence of one hand. Brain I: 388, 1879.

8. GUTHRIE, L. - Muscular atrophy and other changes in nutrition associated with lesions of the sensory cortex of the brain, with special reference to the possible existence of trophic representation in the postcentral areas. Proc. Roy. Soc. Med. $11: 21,1918$. 
9. HEAD, H. - Studies in Neurology. Hodder and Stoughten, London, 1920, vol. 2, pg. 716.

10. MILLS, C. K.; KEENE, W. W. \& SPILLER, W. G. - Tumor of the superior parietal convolution. J. Nerv. a. Ment. Dis. 27:244, 1990.

11. PATEla - Cit por Steinert, H. 19.

12. PENFIELD, W. \& ROBERTSON, J. S. M. - Growth asymetry due to lesions of the postcentral cerebral cortex. Arch. Neurol. a. Psychiat. (Chicago) 50:405, 1943.

13. PITHA, M. V. - Les atrophies musculaires au cours des lesions du lobe parietal. Rev. Neurol. (Paris) $65: 756,1936$.

14. PITHA, M. V.: MASIN, Z. D. \& POLAK, O. - Quelques considerations pathogeniques et cliniques a propos des troubles trophiques dits centraux et surtout l'amyotrophie. Encephale 46:649, 1957.

15. PONS, P. A. - Enfermelades del Sistema Nervioso Tomo IV, Salvat Editores. Madrid, 1974, pg. 89.

16. PRINCE, M. - Limited area of anesthesia, epileptiform attacks of hemiplegia and early muscular athrophy in a case of brain tumor. J. Nerv. a. Ment. Dis. 33: $698,1906$.

17. RHEIN, J. H. - Parietal tumor. J. Nerv. a. Ment. Dis. 62:333, 1920.

18. SILVERSTEIN, A. - Diagnostic localizing value of muscle atrophy in parietal lobe lesions. Neurology (Minneapolis) 5:304, 1955.

19. STEINERT, H. - Cerebral Muakelatrophie cerebralen Ursprungs. Zentralbl. Neurol. $1: 24,1903$.

20. Van BOGAERT, L. - L'amyotrophie precoce dans les tumeurs du lobe parietal. Paris Med. 2:261, 1933.

21. WINKLEMAN, N. W. \& SILVERSTEIN, A. - Unilateral amyotrophy: its diagnostic importance for cerebral localization. Am. J. Syph. \& Neurol. 19:58, 1935.

Hospital sao Domingos - Rua Frrei Paulino $48-38.100$ Uberaba, MG - Brasil. 